\title{
Mapeo y caracterización de servicios ecosistémicos en fincas cultivadas con plátano, Quindío, Colombia (septiembre 2019)
}

\author{
Mapping and characterization of ecosystem services in farms with plantain crops, \\ Quindío, Colombia (September 2019)
}

\author{
A. J. Botero-Arango iD ; A. Feijoo-Martínez iD ; L. J. Molina-Rico iD ; H. Quintero-Vargas iD \\ DOI: https://doi.org/10.22517/23447214.22731 \\ Artículo de investigación científica y tecnológica
}

\begin{abstract}
The indicators related to the Regulatory, Provisioning and Cultural Ecosystem Services were mapped, based on semistructured interviews of owners and Administrators of 32 farms with banana crops, in Quindío Colombia. Three visits were made per site and ethnographic methods were used as a means of data collection, such as dialogue, approach to farmers and their families and semi-structured interviews. Aerial photographs were taken, in order to effect the division of the lots. In these lots, the geospatial position of the SE indicators was considered. The maps were created by means of the Argis 10.1.1 software, a Geographic Information System (GIS) tool. The combination of the participatory mapping of the indicators associated with the ecosystem services, with the GIS tool and the hierarchical analysis of clusters, allowed the grouping of the farms, facilitating the identification of the lots in which both threats and strengths in the generation of said services are presented.
\end{abstract}

Index Terms - Andes, cultural, mapping farms, musaceae, provisioning, regulation.

Resumen - La manera como los agricultores perciben los SE en los agroecosistemas, permite identificar escenarios de interacciones y brindan información de los vínculos entre los humanos y su entorno, y contribuyen con la indagación de las maneras de reducir los impactos futuros en la sociedad ante los constantes cambios ambientales. Se mapearon los indicadores relacionados con los Servicios Ecosistémicos de Regulación, Aprovisionamiento y Culturales, a partir de entrevistas semiestructuradas de propietarios y administradores de 32 fincas con cultivos de plátano, en Quindío Colombia se realizaron tres visitas por predio y se utilizaron métodos etnográficos como medio de recolección de datos, tales como, el diálogo, acercamiento a los agricultores y sus familias y la entrevista semiestructurada. Se tomaron fotografías aéreas, con el fin de efectuar la división de los

Este manuscrito fue enviado el 28 de octubre de 2019 y aceptado 23 de noviembre 2020 .

A. J. Botero-Arango Ingeniero Industrial egresado de la Universidad Tecnológica de Pereira, Risaralda. Profesor Asistente de la Facultad de Ciencias Empresariales, Universidad Tecnológica de Pereira.

(e-mail: joe@utp.edu.co)

A. Feijoo-Martínez. Doctor en Ciencias Agropecuarias de la Universidad Nacional de Colombia, Actualmente Profesor - Investigador Senior, Titular, Facultad de Ciencias Ambientales, Universidad Tecnológica de Pereira.

(e-mail: afeijoo@utp.edu.co) lotes. En dichos lotes se tuvo en cuenta la posición geoespacial de los indicadores de los SE. Los mapas se crearon por medio del software Argis 10.1.1 una herramienta de Sistema de Información Geográfica (SIG). La combinación del mapeo participativo de los indicadores asociados con los servicios ecosistémicos, con la herramienta SIG y el análisis jerárquico de conglomerados, permitió agrupar las fincas, facilitando la identificación de los lotes en que se presentan tanto amenazas como fortalezas en la generación de dichos servicios ecosistémicos. Las percepciones de los agricultores se pueden representar en mapas, que les permiten visualizar más fácilmente para la toma de decisiones. En la literatura estudiada no se encontró documentación relacionada con el mapeo participativo y la caracterización de los servicios ecosistémicos, dirigido específicamente a fincas con cultivos de plátano, por lo que los aportes de este artículo contribuyen como una primera aproximación real sobre la investigación propuesta.

Palabras claves - Andes, aprovisionamiento, culturales, mapeo fincas, musaceae, regulación.

\section{INTRODUCCIÓN}

$\mathrm{L}$ OS cambios en las prácticas y formas de manejo de los $\boldsymbol{u}_{\text {cultivos, conducen a variaciones en los patrones }}$ climáticos, la incidencia de enfermedades y plagas, compactación de los suelos, los que se reflejan en alteraciones en la producción y en repercusiones en los SE.

El plátano herbáceo monocotiledónea, de la familia Musaceae, originaria del sudeste asiático e introducido a

L. J. Molina-Rico. Licenciatura en Biología, Universidad Pedagógica Nacional, Magister Universidad Nacional de Colombia Sede Bogotá, Doctora en Ciencias Ambientales de la Universidad Tecnológica de Pereira. Actualmente Profesora Asociada Universidad del Quindío.

(e-mail: janneth@uniquindio.edu.co)

H. Quintero-Vargas, nacido el 29 de diciembre de 1949 en Roldanillo, Valle del Cauca. Ingeniero Agrónomo de la Universidad Nacional de Colombia, Sede Palmira. Actualmente Profesor Pensionado de la Universidad Nacional de Colombia. 
nuestro país por los españoles en el siglo XVI [1-3], es componente fundamental en la cultura culinaria y en la generación de empleo e ingresos para los cultivadores. En la canasta familiar colombiana, participa en 33\% en el IPC (Índice de Precios al Consumidor) en el grupo de tubérculos, raíces y plátanos.

El cultivo de plátano ha sido sector tradicional en economía campesina, de seguridad alimentaria y de generación de empleo. El departamento del Quindío es el segundo departamento en producción a nivel nacional, ha disminuido su capacidad productiva por problemas en la fertilidad del suelo y sanidad del cultivo [4].

Colombia, debe aumentar la eficiencia económica y ambiental en el sector rural [5]. En la valoración integral de la biodiversidad y los SE interesan las interacciones entre los componentes sociales y ecológicos [6].

$\mathrm{La}$ interacción con las comunidades de agricultores para desarrollar un diálogo que permite conocer la evolución los SE en las áreas de cultivo. El mapeo pone énfasis en las relaciones espaciales entre las características del paisaje tales como el uso / cobertura de la tierra y su contribución en el bienestar humano [7].

En el mapeo de los SE, los agricultores identifican los beneficios directos e indirectos espacialmente explícitos de los ecosistemas que contribuyen con el bienestar humano y también evalúan la importancia relativa de los servicios prestados. Los indicadores de los SE señalan si se conservan y usan de manera sostenible, para tomar las decisiones estos servicios esenciales están siendo conservados y usados de manera sostenible, para tomar las decisiones con el fin de gestionarlos mejor [8].

Los SE no se han evaluado en las fincas cultivadas de plátano en el Quindío y los mapas no se han cartografiado con la participación de las comunidades, para que ellos se den cuenta de la evolución de los servicios por los manejos que ellos han adelantado.

\section{OBJetivos E HIPÓtesis Del Estudio}

\section{Objetivo general:}

Vincular a los cultivadores de plátano el mapeo de indicadores relacionados con servicios ecosistémicos que les ayuden a detectar puntos críticos para el manejo sostenible de sus fincas.

Objetivos específicos:

Indagar las percepciones de los cultivadores acerca de los SE de Regulación, Aprovisionamiento y Culturales, en 32 fincas de un área del departamento del Quindío.

Combinar la cartografía social de los SE de Regulación, Aprovisionamiento y Culturales con el análisis exploratorio de la información.

Consolidar líneas de acción que posibiliten fortalecer la toma de decisiones de los agricultores.

A partir de lo anterior se propuso como hipótesis:

Es posible vincular a los cultivadores de plátano en determinar las condiciones de los lotes en las fincas por medio de la utilización del mapeo de los servicios ecosistémicos.

\section{Metodología}

\section{A. Área de estudio}

El trabajo se llevó a cabo en 32 fincas de los municipios de Armenia, Calarcá y Circasia (Fig. 1), con un área total de 296 hectáreas en donde el principal sistema productivo es el cultivo de plátano de la variedad Dominico Hartón (DH), y que se siembra en uno, dos o tres lotes por finca. Las fincas se encuentran dentro de la cuenca del río La Vieja; en el piso térmico subandino, desde los 1197 hasta los 1588 m.s.n.m. La temperatura oscila entre los $18^{\circ} \mathrm{C}$ y $24^{\circ} \mathrm{C}$ y la precipitación media anual entre $2000 \mathrm{~mm}$ y $2200 \mathrm{~mm}$ (IGAC. 2014).

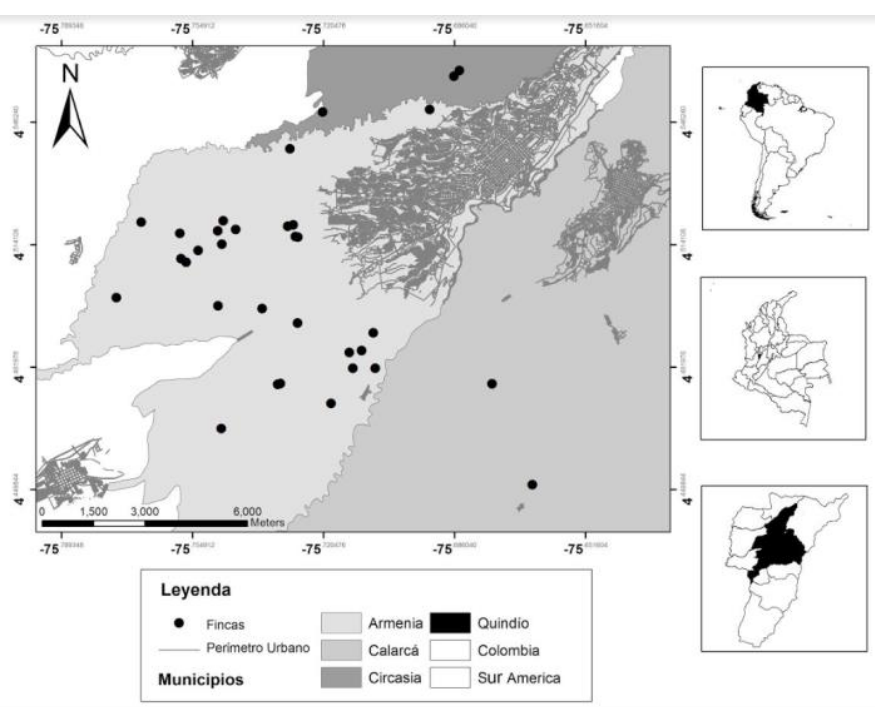

Fig. 1. Situación de las fincas en tres municipios del departamento del Quindío. Fuente: GATA.

La información se obtuvo dentro del macro-proyecto "Servicios ecosistémicos generados por diversos arreglos del cultivo de plátano en el Eje Cafetero Colombiano" del grupo de investigación en Gestión de Agroecosistemas Tropicales Andinos (GATA) de la Universidad Tecnológica de Pereira. En cada finca se realizaron tres visitas, se indagó acerca de las percepciones y se utilizaron métodos etnográficos como medio de recolección de datos, tales como, el diálogo, acercamiento a los agricultores y sus familias y la entrevista semiestructurada [9], en las que se consultó acerca del nivel educativo, la procedencia y el tiempo de permanencia en años en la finca. En cuatro talleres de intercambio, en los que se discutió personalmente la información de cada predio, con la finalidad de reforzar el conocimiento local de los agricultores. Además, para ampliar las percepciones de los cultivadores de plátano, se tuvo en cuenta la posición geoespacial de los indicadores de los SE por lote y se usó la información de fotografías satelitales y de dron de las fincas. En cada uno de los mapas se identificó la división de los lotes por finca en los que se podía dibujar, marcar con letras la representación de cada pregunta y poner 
colores que demarcaban los límites y las variantes por cada predio.

En el intercambio con los agricultores, se programó el segundo taller titulado "Relatos compartidos para mapear SE en cultivos de plátano en fincas del Quindío, Colombia" Para realizar el mapeo de los indicadores asociados con los SE de Regulación se realizaron siete preguntas: ¿En qué lugar de la finca el suelo es más compacto y polvoriento? (C), ¿Cuáles lotes presentan problemas de encharcamiento? (E), ¿En cuáles lotes hay mayor presencia de humedad? (H), ¿En qué lotes se presenta la mayor presencia de vendavales? $(\mathrm{V})$, ¿En cuales lotes hay mayor presencia de plagas? (G), ¿En cuáles lotes se observa mayor presencia de picudo $(\mathrm{Q})$, ¿En cuáles lotes hay mayor presencia de enfermedades? (F). Para comprender cómo el agricultor percibía los indicadores relacionados con los SE de aprovisionamiento se formularon cuatro preguntas: ¿Dónde prefiere sembrar plátano? (S) ¿Cuáles lotes producen más plátano? (P) ¿Cuáles lotes producen racimos más grandes? (R) ¿En cuáles lotes se observa mayor presencia de lombrices, escarabajos, tijeretas? (M). Adicionalmente, para interpretar los valores de importancia cultural que les conceden los agricultores a las partes de las fincas, se formularon tres preguntas que indagaron acerca de: ¿Con qué lugares de su finca se sienten identificados? (L) ¿Cuál fue la forma de adquisición de la finca? (O) ¿Cuáles son los lugares que simbolizan arraigo o apego a su entorno? (A).

\section{B. Análisis espacial}

En los mapas de las fotografías aéreas, se demarcaron los lotes y se identificaron los indicadores relacionados con los SE. Los mapas se crearon por medio del software Argis 10.1.1, herramienta del Sistema de Información Geográfica (SIG). El instrumento permitió cargar la plataforma Bing Aerial, que aloja imágenes en la nube -internet-. Estas herramientas permitieron almacenar, transformar, analizar, modelar y mapear fenómenos y procesos que caracterizan el espacio geográfico [10]. Se tomaron fotografías con un Dron, para que los agricultores identificaran los lugares donde existían los indicadores de los SE en sus fincas. Se indicaron las coordenadas geográficas de cada finca y se ingresaron al software para visualizar los predios en la imagen satelital. Con el software Argis se creó una capa (Mecanismo de visualización de datos geográficos) en forma de un polígono, representando el contorno de cada finca como lo dibujó el agricultor previamente en la fotografía, luego se generó dentro del polígono una nueva capa con las divisiones de los lotes, los cuales se digitalizaron. Posteriormente, se crearon polígonos y círculos en la capa de lotes, los cuales representan los indicadores de los SE asociados con lugares específicos de la finca; se les asignó un ID con nombre y color, azul para los de Regulación (S.E.R), verde para los de Aprovisionamiento (S.E.A) y amarillo para los Culturales (S.E.C). En los casos de las fincas donde los agricultores manifestaban que cierto indicador se presentaba en toda la finca se procedió a generar un polígono que agrupara todos los lotes y se les asignó el color correspondiente al SE que representaba con un diseño de grabado específico y con transparencia del polígono para permitir la visualización de la imagen satelital del predio. Cada capa se exportó al formato Shapefile, los mapas se generaron en WCS_WGS_1984 que permite localizar cualquier punto de la tierra, realizadas con escalas detalladas comprendidas entre 1:300 y 1:5000 de las fincas. (Fig. 2).

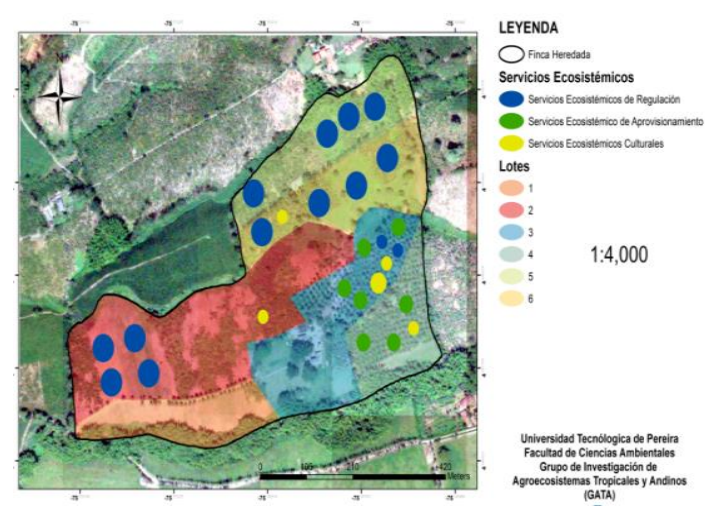

Fig. 2. Ejemplo mapeo indicadores de los servicios ecosistémicos en una de las fincas.

\section{Análisis estadístico}

Para llevar a cabo el análisis exploratorio se digitalizó la información obtenida de los agricultores, separando la información cualitativa, en el programa Excel 2013. Para estimar los datos faltantes, se utilizó la media en el caso de los indicadores relacionados con los S.E.R (Servicios Ecosistémicos de Regulación) y los S.E.A (Servicios Ecosistémicos de Aprovisionamiento) y la moda para los indicadores de los S.E.C (Servicios Ecosistémicos culturales). Los datos de entrada para el análisis de conglomerados jerárquicos realizado con el software SSPS Versión 22, fueron el porcentaje de lotes en los cuales se evidenció la presencia de cada uno de los indicadores. En la parametrización del programa se tomó como medida de distancia, la euclideana al cuadrado, y como método de agrupamiento el Método de Ward (o método de pérdida de la inercia mínima), que une los casos buscando minimizar la varianza dentro de cada grupo. El criterio de corte utilizado fue del $25 \%$ (correspondiente a 6,25 en la escala de distancias) dado que este valor muestra un buen nivel de discriminación y agrupamiento de las fincas.

Para efecto del análisis de conglomerados jerárquicos, los S.E.R se clasificaron [11] en: (i) SE Regeneración y Formación del suelo, conformado por los indicadores: número de lotes con el suelo compacto y polvoriento, encharcamiento y presencia de humedad; (ii) SE Regulación climática, representado por el indicador: número de lotes con mayor presencia de vendavales y; (iii) SE Regulación de las enfermedades, relacionado con los indicadores: número de lotes con mayor presencia de picudo, con mayor presencia plagas y con mayor presencia de enfermedades.

Los S.E.A, se clasificaron [11] en: (i) SE Producción de alimentos, conformado por los indicadores: número de lotes donde el agricultor prefiere sembrar, donde se da la mayor 
producción y los racimos más grandes; (ii) SE Actividad biológica, representado por el indicador: número de lotes con mayor presencia de lombrices, escarabajos y tijeretas. Como primer paso se generó el conglomerado de pertenencia, al que se asigna cada finca y el correspondiente Dendrograma. Para el análisis descriptivo, en el cual se eligieron los siguientes estadísticos univariantes: El promedio del \% de lotes $(\bar{x})$ y la desviación típica (s), que permiten la caracterización de las fincas asociadas en cada conglomerado.

En el caso de los S.E.C por tratarse de variables cualitativas, se realizó el análisis por medio de tablas de contingencia $(2 \times 2)$, para lo cual se realizaron dos tabulaciones: en la primera se cruzaron los indicadores: lotes con los que se identifica y modos de adquisición de la finca, y en la segunda se analizaron los indicadores: lugares que simbolizan arraigo y modos de adquisición de la finca, utilizando la Prueba Ji- cuadrado de Pearson. Se contrastó la hipótesis nula Ho: las dos variables analizadas son independientes, con un nivel de significancia de $\alpha=0,05$, Si el p-valor asociado al estadístico es menor que $\alpha$ se rechazará la hipótesis nula en el caso contrario se aceptará.

\section{RESUlTADOS}

\section{A. Caracterización social de los agricultores}

Han cursado la primaria, 11 (35\%) de los propietarios y administradores mientras que 10 (28\%) realizaron estudios de bachillerato incompleto, en tanto que tres (9\%), finalizaron la educación secundaria y media, y tres $(9 \%)$ tuvieron formación tecnológica; en el caso de la formación superior, se encontró que cinco (19\%) y uno (3\%) concluyeron el pregrado y posgrado respectivamente. En el caso de la procedencia, se encontró que el $66 \%$ provenían de los departamentos del Eje Cafetero, siendo la mayor participación el departamento del Quindío con un 53\%, en tanto los restantes (34\%) llegaron a la región de Antioquia, Valle, Cundinamarca, Tolima y Cauca. Al analizar el tiempo de permanencia $16 \%$ de los propietarios han administrado las fincas durante más de 40 años, mientras que un $37 \%$ han permanecido entre 10 y 40 años, y el $47 \%$ restante por menos de 10 años. El 87\% laboran en las actividades correspondientes a los cultivos de plátano y otras plantaciones según el caso, en un día normal, entre 3 y 11 horas, y el restante $13 \%$ les dedican a estos cultivos menos de cinco horas al día (Tabla I).

TABLA I

DESCRIPTORES SOCIALES DE LOS AGRICULTORES EN LAS 32 FINCAS DEL DEPARTAMENTO DEL QUINDÍO

\begin{tabular}{|c|c|c|c|c|c|c|}
\hline $\begin{array}{l}\text { No. } \\
\text { De } \\
\text { finc } \\
\text { a } \\
\end{array}$ & $\begin{array}{c}\text { Municip } \\
\text { io }\end{array}$ & $\begin{array}{c}\text { Nivel } \\
\text { Educativ } \\
0\end{array}$ & $\begin{array}{c}\text { Procedenc } \\
\text { ia }\end{array}$ & $\begin{array}{c}\text { Tiempo de } \\
\text { permanencia } \\
\text { en años } \\
(2016)\end{array}$ & Función & $\begin{array}{l}\text { Hora } \\
\text { s } \\
\text { labor } \\
\text { ables }\end{array}$ \\
\hline 1 & Armenia & $\begin{array}{c}\text { Bachillera } \\
\text { to }\end{array}$ & Caldas & 40 & $\begin{array}{c}\text { PROP- H- } \\
\text { TRAB }\end{array}$ & 9 \\
\hline 2 & Armenia & Primaría & Quindío & 17 & ADM-TRAB & 8 \\
\hline 3 & Armenia & $\begin{array}{c}\text { Bachillera } \\
\text { to } \\
\text { Incomplet } \\
0\end{array}$ & Antioquía & 26 & PROP & 11 \\
\hline
\end{tabular}

\begin{tabular}{|c|c|c|c|c|c|c|}
\hline 4 & Armenia & $\begin{array}{c}\text { Bachillera } \\
\text { to } \\
\text { Incomplet } \\
0\end{array}$ & Valle & $\overline{116}$ & 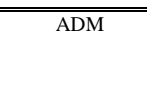 & 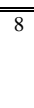 \\
\hline 5 & Armenia & Primaría & Quindío & 20 & TRAB & 8 \\
\hline 6 & Armenia & Primaría & Caldas & 10 & TRAB & 8 \\
\hline 7 & Calarca & Primaría & Quindío & 7 & PROP & 10 \\
\hline 8 & Calarca & Primaría & Risaralda & 16 & $\begin{array}{l}\text { PROP- H- } \\
\text { TRAB }\end{array}$ & 10 \\
\hline 9 & Armenia & Primaría & Quindío & 3 & ADM & 9 \\
\hline 10 & Armenia & $\begin{array}{c}\text { Bachillera } \\
\text { to } \\
\text { Incomplet }\end{array}$ & $\mathrm{C} / \mathrm{marca}$ & 8 & PROP & 9 \\
\hline 11 & Armenia & $\begin{array}{c}\text { Bachillera } \\
\text { to } \\
\text { Incomplet } \\
0\end{array}$ & Tolima & 68 & PROP & 9 \\
\hline 12 & Armenia & $\begin{array}{l}\text { Profesion } \\
\text { al }\end{array}$ & Antioquía & 21 & TRAB & 4 \\
\hline 13 & Armenia & $\begin{array}{c}\text { Tecnólog } \\
\text { o }\end{array}$ & Quindío & 50 & PROP-TRAB & 9 \\
\hline 14 & Armenia & $\begin{array}{c}\text { Bachillera } \\
\text { to }\end{array}$ & Quindío & 13 & PROP-TRAB & 4 \\
\hline 15 & Armenia & $\begin{array}{c}\text { Tecnólog } \\
\text { o }\end{array}$ & Valle & 2 & TRAB & 8 \\
\hline 16 & Armenia & $\begin{array}{c}\text { Tecnólog } \\
\text { o }\end{array}$ & Valle & 2 & PROP- TRAB & 8 \\
\hline 17 & Armenia & $\begin{array}{c}\text { Bachillera } \\
\text { to } \\
\text { Incomplet } \\
\text { o }\end{array}$ & Antioquía & 2,3 & $\begin{array}{l}\text { ADMI-H- } \\
\text { TRAB }\end{array}$ & 8 \\
\hline 18 & Armenia & Primaría & Risaralda & 5 & PROP- TRAB & 8 \\
\hline 19 & Armenia & $\begin{array}{c}\text { Bachillera } \\
\text { to } \\
\text { Incomplet } \\
0\end{array}$ & Quindío & 4 & ADM-, TRAB & 8 \\
\hline 20 & Circasia & Postgrado & Quindío & 14 & TRAB & 9 \\
\hline 21 & Armenia & $\begin{array}{c}\text { Profesion } \\
\text { al }\end{array}$ & Quindío & 13 & TRAB & 8 \\
\hline 22 & Armenia & $\begin{array}{c}\text { Bachillera } \\
\text { to } \\
\text { Incomplet } \\
0\end{array}$ & Antioquía & 50 & PROP & 5 \\
\hline 23 & Circasia & $\begin{array}{l}\text { Profesion } \\
\quad \text { al }\end{array}$ & Quindío & 3 & TRAB & \\
\hline 24 & Armenia & $\begin{array}{l}\text { Profesion } \\
\text { al }\end{array}$ & Quindío & 1 & $\mathrm{ADM}$ & 9 \\
\hline 25 & Armenia & $\begin{array}{c}\text { Bachillera } \\
\text { to } \\
\text { Incomplet } \\
0\end{array}$ & Quindío & 5 & $\mathrm{ADM}$ & 9 \\
\hline 26 & Armenia & $\begin{array}{l}\text { Profesion } \\
\quad \text { al }\end{array}$ & Quindío & 10 & ADM- TRAB & 9 \\
\hline 27 & Circasia & $\begin{array}{c}\text { Bachillera } \\
\text { to }\end{array}$ & Quindío & 0,2 & ADM, TRAB & 9 \\
\hline 28 & Armenia & Primaría & Antioquía & 45 & PROP- TRAB & 9 \\
\hline 29 & Armenia & Primaría & Cauca & 3 & ADM, TRAB & 3 \\
\hline 30 & Armenia & Primaría & Quindío & 2,6 & ADM & 8 \\
\hline 31 & Armenia & Primaria & Quindío & 1,5 & ADM & 8 \\
\hline 32 & Armenia & $\begin{array}{c}\text { Bachillera } \\
\text { to } \\
\text { Incomplet } \\
0\end{array}$ & Quindío & 20 & ADM & 9 \\
\hline
\end{tabular}

$\mathrm{PROP}=$ propietario; $\mathrm{ADM}=$ administrador; TRAB=trabajador; $\mathrm{H}=$ hijo

\section{B. Servicios Ecosistémicos}

Para el SE Regeneración y Formación del suelo se agruparon las fincas en tres conglomerados: el primero conformado por 26 fincas $(1,3,4,5,6,7,8,9,10,11,12,13,14,16,18,19,20,21,24$, $25,26,27,28,29,30,31,32$ ) que representan el $81 \%$, y se caracterizaron por presentar el porcentaje promedio de lotes más bajos, alrededor del 26\% para sus indicadores analizados: suelo compacto y polvoriento, encharcamiento, y presencia de 
humedad. El segundo asoció $12.5 \%$ de las fincas $(2,15,23$, 30 ), cuyo porcentaje promedio de lotes para los tres indicadores fue del 50\%. El tercero agrupó solamente dos fincas $(17,22)$ con la representación más baja del $6,2 \%$, pero que se caracterizaron por tener el porcentaje más bajo del primer indicador, del $16 \%$, pero a su vez el porcentaje más alto, el $100 \%$ de los otros dos indicadores (Fig. 3 y Tabla II).

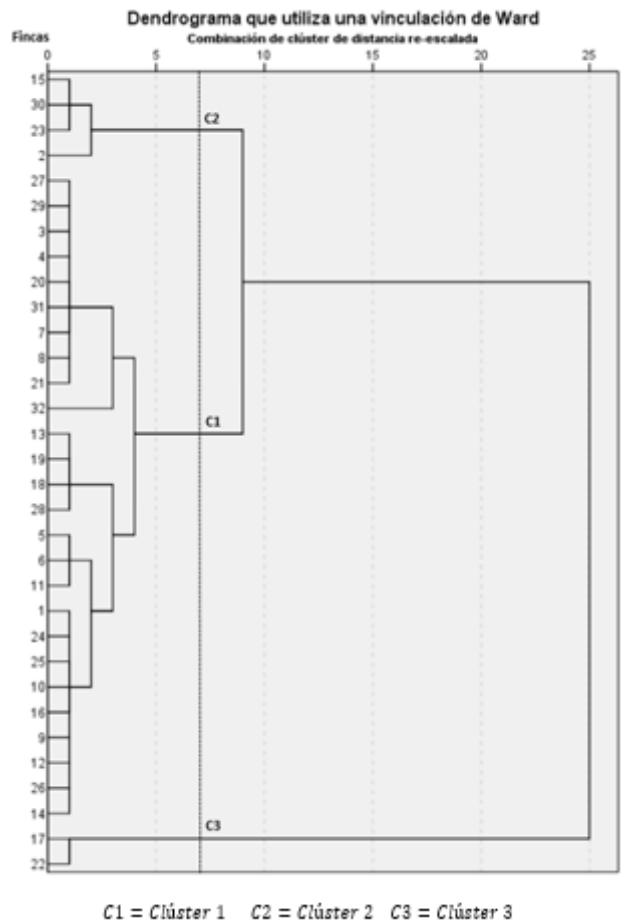

Fig. 3. Dendrograma agrupación de fincas según indicadores SE Regeneración y Formación del suelo. Fuente SSPS versión 22

TABLA II.

PARÁMETROS INDICADORES SE REGULACIÓN Y FORMACIÓN DEL SUELO. FUENTE: SSPS VERSIÓN 22

\begin{tabular}{|c|c|c|c|c|}
\hline Grupo & Parámetros & $\begin{array}{l}\% \text { de suelo } \\
\text { compacto }\end{array}$ & $\begin{array}{c}\% \text { de } \\
\text { encharcamiento }\end{array}$ & $\begin{array}{c}\% \\
\text { presencia } \\
\text { de } \\
\text { humedad }\end{array}$ \\
\hline \multirow[t]{2}{*}{1} & Media & 23,4 & 26,6 & 29,5 \\
\hline & $\begin{array}{l}\text { Desviación } \\
\text { estándar }\end{array}$ & 11,4 & 8,3 & 6,2 \\
\hline \multirow[t]{2}{*}{2} & Media & 48,0 & 50,0 & 50,0 \\
\hline & $\begin{array}{l}\text { Desviación } \\
\text { estándar }\end{array}$ & 17,1 & 13,6 & 13,6 \\
\hline \multirow[t]{2}{*}{3} & Media & 16,7 & 100,0 & 100,0 \\
\hline & $\begin{array}{l}\text { Desviación } \\
\text { estándar }\end{array}$ & 23,6 & 0 & , 0 \\
\hline
\end{tabular}

Para el SE Regulación Climática, las fincas se asociaron en tres categorías, la primera consta de seis fincas $(1,2,12,21,23$, 30) que representan el $18,8 \%$, en las cuales el porcentaje promedio de presencia de vendavales arrojó el resultado más alto con un $83 \%$, en la segunda categoría se agruparon 15 fincas $(3,4,5,6,8,15,16,17,19,20,26,27,28,29,32)$ con una participación del 46,9\%, en las cuales este indicador representó un $40 \%$, y en la tercera categoría 11 fincas $(7,9,10,11,13,14$, $18,22,24,25,31)$ con una participación equivalente al $34,4 \%$, en las cuales la presencia de este indicador fue las más baja del $15 \%$ (Fig. 4, Tabla III).

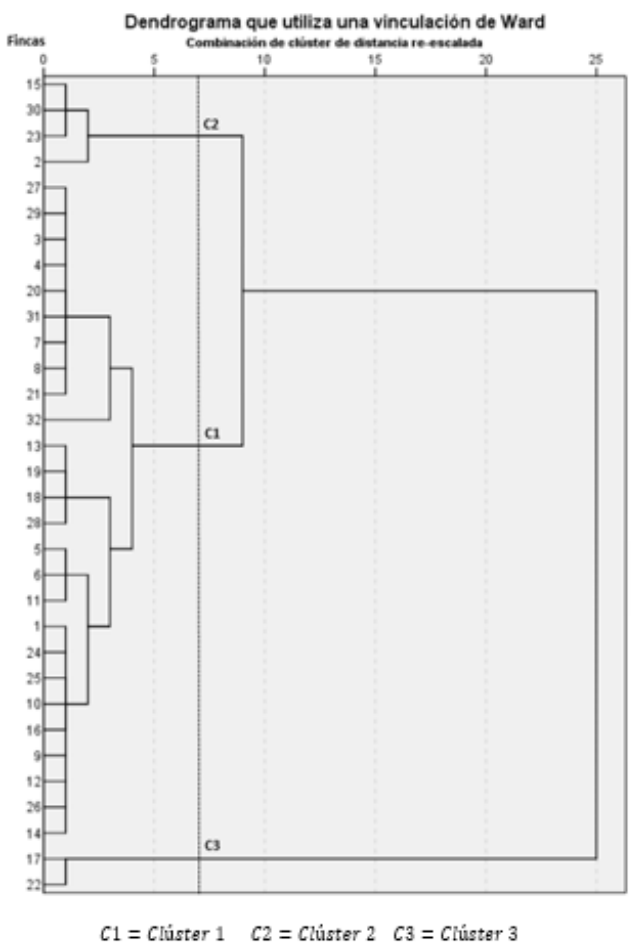

Fig. 4. Dendrograma para la agrupación de fincas según el indicador Regulación Climática. Fuente SSPS versión 22

TABLA III.

Parámetros Indicadores SE Regulación Climática. Fuente SSPS VERSIÓN 22

\begin{tabular}{ccc}
\hline \hline Grupo & Media & Desviación Estándar \\
\hline $\mathbf{1}$ & 83,2 & 15,0 \\
$\mathbf{2}$ & 39,7 & 7,2 \\
$\mathbf{3}$ & 15,1 & 11,0 \\
\hline \hline
\end{tabular}

En el SE Regulación de las Enfermedades, evaluado por los indicadores presencia de picudo, de plagas y de enfermedades, las fincas se agruparon en cuatro categorías. La primera conformada por siete fincas $(1,2,10,13,23,27,30)$ que equivalen al $21,9 \%$, en las cuales el porcentaje de lotes con presencia del primer indicador fue de los más altos el $94 \%$, mientras los otros dos indicadores fueron del $38 \%$ y del $22 \%$. La segunda agrupó 16 fincas $(3,4,5,8,9,12,19,20,21,22$, $24,25,26,28,29,31)$ con una participación del $50 \%$, las que 
se caracterizaron por que sus porcentajes para los 3 indicadores fueron alrededor del $36 \%$.

La tercera la conformaron siete fincas $(6,11,14,15,16,18$, 32 ) que representan el $21,9 \%$., en las que a pesar de presentar el porcentaje más bajo de presencia de picudo $26 \%$, presentó unos porcentajes más altos de presencia de plagas $74 \%$ y presencia de enfermedades $69 \%$. En la cuarta categoría se asociaron solamente dos fincas $(7,17)$ con la representación más baja del 6,3\%, pero donde se da la mayor presencia de picudo y enfermedades del 100\% y de plagas del 74\% (Fig. 5, Tabla IV).

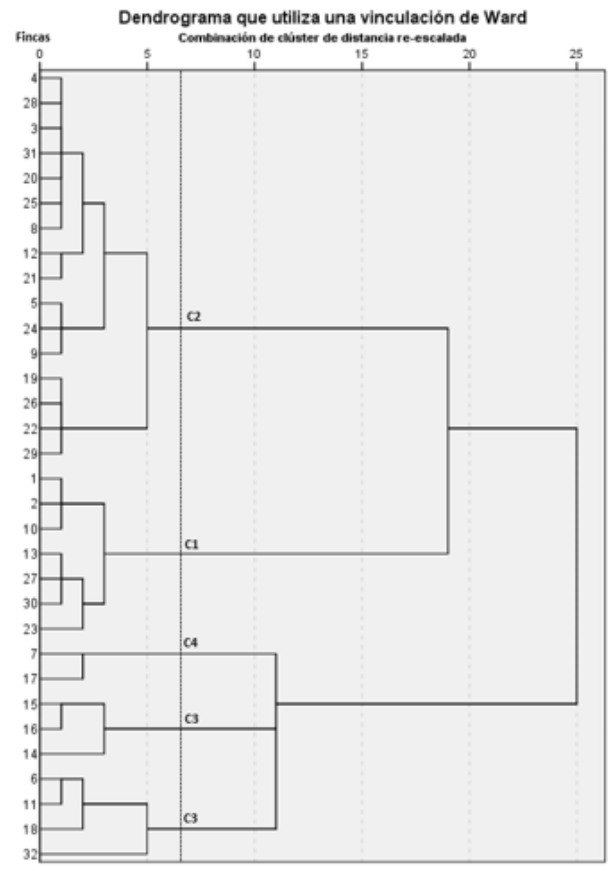

$C 1=$ Clúster $1 \quad C 2=$ Clúster $2 \quad C 3=$ Clúster $3 \quad C 4=$ Clúster 4

TABLA IV.

PARÁMETROS INDICADORES SE REGULACIÓN FUENTE SSPS VERSIÓN 22

\begin{tabular}{llrrr}
\hline \hline Grupo & Parámetros & $\begin{array}{c}\text { \% con } \\
\text { mayor } \\
\text { presencia } \\
\text { de picudo }\end{array}$ & $\begin{array}{c}\text { \% con mayor } \\
\text { presencia de } \\
\text { plagas }\end{array}$ & $\begin{array}{c}\text { \% mayor } \\
\text { presencia de } \\
\text { enfermedades }\end{array}$ \\
\hline $\mathbf{1}$ & Media & 94,4 & 38,4 & 22,8 \\
& $\begin{array}{l}\text { Desviación } \\
\text { estándar }\end{array}$ & 10 & 19,8 & 15,8 \\
$\mathbf{2}$ & Media & 36,6 & 38,5 & 33,3 \\
& $\begin{array}{l}\text { Desviación } \\
\text { estándar }\end{array}$ & 18,3 & 14,7 & 12,4 \\
$\mathbf{3}$ & $\begin{array}{l}\text { Media } \\
\text { Desviación }\end{array}$ & 18,7 & 74,8 & 69,4 \\
$\mathbf{4}$ & estándar & 100 & 38,3 & 34,1 \\
\hline \hline
\end{tabular}

\begin{tabular}{cccc}
\hline \hline $\begin{array}{l}\text { Desviación } \\
\text { estándar }\end{array}$ & 0 & 36,2 & 0 \\
\hline \hline
\end{tabular}

Los SEA se clasificaron de acuerdo con MEA (2005) en los siguientes: (i) SE Producción de alimentos, relacionados con los siguientes tres indicadores: preferencia para sembrar, mayor producción y racimos más grandes. (ii) SE Actividad biológica relacionada con el indicador mayor presencia de lombrices, escarabajos y tijeretas. Para el SE Producción de alimentos, se agruparon las fincas en cuatro conglomerados. El primero comprende 17 fincas $(1,5,8,10,12,14,18,19,21,23,24,25$, $26,27,28,29,31)$ que representan más de la mitad de las fincas con el 53,1\%; caracterizadas por presentar los porcentajes promedios de lotes más bajos con la presencia de estos tres indicadores: $35 \%$ con preferencia para sembrar, y $27 \%$ con mayor producción y racimos más grandes. El segundo lo conforman ocho fincas, $(2,3,4,6,7,15,20,30)$ con una participación del $25 \%$; en las cuales la presencia de estos tres indicadores corresponde con el porcentaje promedio del $50 \%$. En el tercer conglomerado se asociaron solamente dos fincas ( 9 , 16), que equivalen al 6,2\%; pero con el porcentaje de presencia de los tres indicadores más alto del $100 \%$. En el último se agruparon cinco fincas $(11,13,17,22,32)$ con una representación del $15,6 \%$ caracterizada porque presentan un buen porcentaje promedio de lotes $92 \%$, con relación al primer indicador, pero solamente de un $40 \%$ respecto a los otros dos indicadores (Fig. 6, Tabla V).

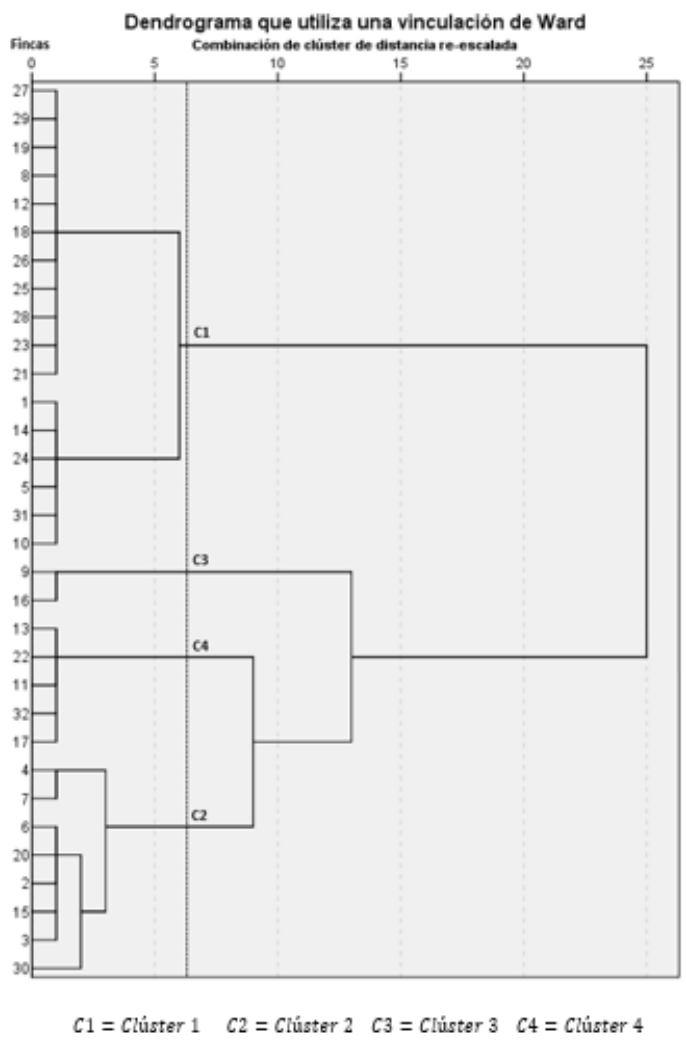

Fig. 1. Dendrograma agrupación de fincas según indicadores SE Producción de Alimentos Fuente SPSS versión 22 
TABLA V.

PARÁmetros IndicAdores SE Producción de Alimentos. Fuente SSPS VERSIÓN 22

\begin{tabular}{|c|c|c|c|c|}
\hline Grupo & Parámetros & $\begin{array}{c}\% \text { de } \\
\text { preferencia } \\
\text { para } \\
\text { sembrar }\end{array}$ & $\begin{array}{c}\% \text { de lotes con } \\
\text { mayor } \\
\text { producción }\end{array}$ & $\begin{array}{l}\% \text { de lotes } \\
\text { con racimos } \\
\text { más grandes }\end{array}$ \\
\hline \multirow[t]{2}{*}{1} & Media & 35,5 & 27,5 & 27,0 \\
\hline & $\begin{array}{l}\text { Desviación } \\
\text { estándar }\end{array}$ & 16,7 & 8,7 & 10,9 \\
\hline \multirow[t]{2}{*}{2} & Media & 49,8 & 43,2 & 65,0 \\
\hline & $\begin{array}{l}\text { Desviación } \\
\text { estándar }\end{array}$ & 16,2 & 14,0 & 15,7 \\
\hline \multirow[t]{2}{*}{3} & Media & 100 & 100 & 100 \\
\hline & $\begin{array}{l}\text { Desviación } \\
\text { estándar }\end{array}$ & 0 & 0 & 0 \\
\hline \multirow[t]{2}{*}{4} & Media & 92,1 & 40,0 & 39,2 \\
\hline & $\begin{array}{l}\text { Desviación } \\
\text { estándar }\end{array}$ & 11,4 & 9,7 & 12,8 \\
\hline
\end{tabular}

Para el SE Actividad Biológica se agruparon las fincas en dos categorías. La primera conformada por 21 fincas $(1,2,3,4,5$ ,6, 7, 10, 11, 13, 14, 15, 16, 18, 19, 23, 24, 25, 26, 31, 32) que representan el $65,6 \%$, caracterizadas por presentar el porcentaje promedio de lotes del $35 \%$, con presencia de lombrices, escarabajos y tijeretas. En la segunda se agruparon 11 fincas $(8$, $9,12,17,20,21,22,27,28,2930$ ), que equivalen al 34,4\%, con la participación más alta del indicador en el $100 \%$ de los lotes. (Fig. 7, Tabla VI).

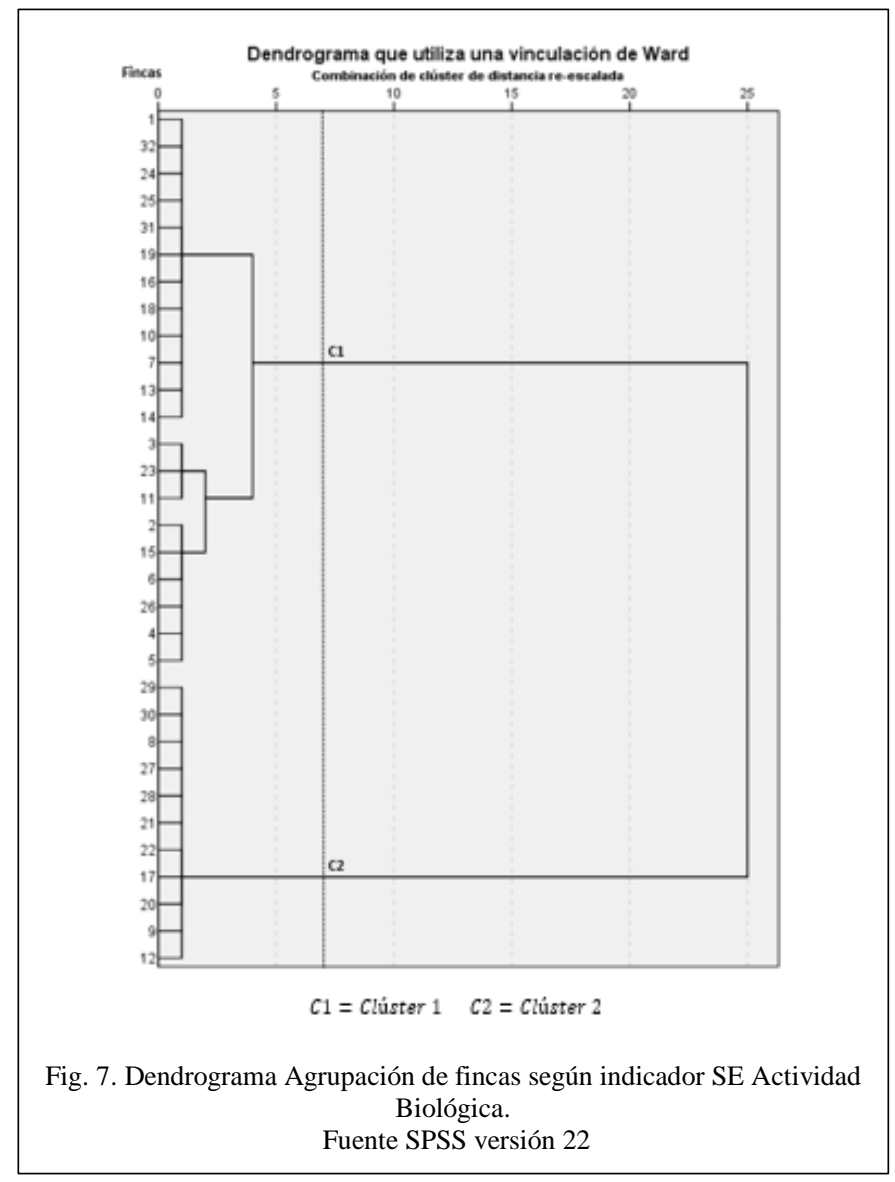

TABLA VI.

PARÁMETROS INDICADOR SE ACTIVIDAD BIOLÓGICA: PRESENCIA DE LOMBRICES, ESCARABAJOS Y TIJERETAS. FUENTE SPSS VERSIÓN 22.

\begin{tabular}{ccc}
\hline \hline Grupo & Media & Desviación Estándar \\
\hline $\mathbf{1}$ & 34,9 & 9,9 \\
$\mathbf{2}$ & 100 & 0 \\
\hline \hline
\end{tabular}

En el caso de los SE culturales, con la tabla de contingencia se pretende evaluar el grado de relación existente entre las dos variables categóricas (lotes con los que se identifica y modos de adquisición de la finca) y el estadístico Ji - cuadrado, lo cual permite contrastar la hipótesis de que los dos criterios de clasificación utilizados son independientes.

La probabilidad obtenida de 0,805 es mayor que la significancia asumida $(\alpha=0,05)$ por lo cual se acepta la hipótesis de independencia y se concluye que los lotes con los que se identifica y los modos de adquisición de la finca no están relacionados

Del total de los agricultores (24) que heredaron las fincas, se identifican con un lugar específico, como, por ejemplo, el nacimiento de agua, el huerto, la casa, el salón social etc, un total de 19 , que equivalen al 79,2\%. Los cinco restantes que representan el 20,8\%, se identifican con toda la finca. Del total de agricultores (8) que compraron las fincas, se identifican con un lugar específico seis que equivalen al 75\%. Los otros dos, que representan el $25 \%$, se identifican con toda la finca.

En el caso de los indicadores lugares que simbolizan arraigo y modos de adquisición de la finca la probabilidad obtenida de 0,346 es mayor que la significancia asumida $(\alpha=0,05)$ por lo cual se acepta la hipótesis de independencia y se concluye que los lugares que simbolizan arraigo y los modos de adquisición de la finca no están relacionados.

Del total de los agricultores (24) que heredaron las fincas, sienten arraigo por toda la finca un total de 19 , que equivalen al $79,2 \%$, mientras que los cinco restantes que representan el $20,8 \%$, sienten arraigo por lugares diferentes, tales como, la escuela, la vereda, el colegio, el comité de cafeteros etc. Del total de agricultores (8) que compraron las fincas, sienten arraigo por otros lugares, seis, que equivalen al $75 \%$, los dos restantes, que representan el $25 \%$, sienten arraigo por toda la finca.

\section{DISCUSIÓN}

\section{A. El valor de la participación de los agricultores a partir de las percepciones}

El nivel de educación formal de la mayoría de propietarios y/o administradores fue bajo, lo que implica que apoyan sus prácticas agrícolas en saberes tradicionales, transmitidos de los antepasados. La mayoría procedía del Eje Cafetero, sobresaliendo los del Departamento del Quindío; que han 
permanecido por más de 40 años, lo cual indica el conocimiento que tienen acerca del aprovechamiento de las fincas.

Un desafío que enfrentan tanto agricultores, como investigadores es saber ¿cuándo un agroecosistema se puede considerar saludable?, o ¿En qué estado de salud se encuentra? Los investigadores que trabajan en agricultura sostenible han propuesto una serie de indicadores de sostenibilidad para evaluar el estado de los agroecosistemas con cultivos [12]. Algunos indicadores consisten en observaciones o mediciones que se realizan a nivel de finca para determinar la fertilidad y conservación del suelo y si las plantas están sanas, vigorosas y productivas.

En la mayoría de los casos, los mapas obtenidos por medio de estudios participativos, en los que intervienen directamente los agricultores, son de mayor calidad y más relevantes que los producidos por agentes externos [13], de ahí radica la importancia del presente estudio, ya que el mapeo se realizó con la participación de los agricultores, pilar fundamental, ya que son los que poseen directamente el conocimiento de sus fincas, utilizando para ello el mapeo participativo, que hizo posible identificar en cada uno de los lotes la presencia de los diferentes indicadores asociados con los SE estudiados, que permitieron el agrupamiento de acuerdo con sus semejanzas por medio del análisis de conglomerados.

La identificación de los indicadores de los Servicios Ecosistémicos prestados por las fincas, utilizando la cartografía social, es una herramienta de gran utilidad para generar sensibilidad, aprender e intercambiar conocimiento científico y local con los agricultores [14] e impulsar acciones de conservación o restauración del ecosistema, así como de prevención de daños adicionales que puedan perjudicar dichos ecosistemas [15].

Quizás lo más importante es que una vez aplicados los indicadores, cada agricultor visualiza el estado de su finca, observando los atributos del suelo o del cultivo que tienden a la recuperación o degradación en relación con umbrales preestablecidos, pero potenciados o afectados por la manera como se maneja el cultivo [16].

La implementación del mapeo participativo y la caracterización de los Servicios Ecosistémicos de Regulación, Aprovisionamiento y Culturales, permitió a los agricultores identificar en sus fincas, aquellos lotes en los cuales se presentan procesos de degradación, con el fin de tomar decisiones encaminadas a mejorar el uso del suelo, como por ejemplo diversificar con otro tipo de agricultura.

\section{B. Indicadores de los SE de Regulación, Aprovisionamiento y Culturales con el análisis exploratorio de la información.}

La caracterización por medio de indicadores en sistemas de cultivos de plátano, facilita la medición y el seguimiento de las prácticas agroecológicas a nivel de fincas. Por lo tanto, es necesario llevar a cabo esta actividad bajo los mismos criterios de valoración cualitativos o cuantitativos para que los resultados sean comparables, de tal forma que se pueda seguir la trayectoria de un mismo agroecosistema a través del tiempo, o realizar comparaciones entre fincas en varios estados de transición [17].

Un sistema más diversificado con rizosfera abundante y diferente y un suelo rico en materia orgánica y biológicamente activo es considerado un sistema no degradado, robusto y productivo. En otras palabras, un agroecosistema con un cultivo prevalente rico en biodiversidad, la cual a partir de una serie de sinergismos contribuye con la fertilidad edáfica, la fitoprotección y la productividad del sistema, se considera sustentable o saludable [18].

El desarrollo y uso de indicadores, resulta una herramienta adecuada y flexible para evaluar tendencias, establecer diferencias entre fincas y detectar los puntos críticos de manejo de recursos para el logro de una agricultura sustentable [19]. Esto es útil para que los agricultores entiendan porqué ciertas fincas se comportan mejor que otras, y qué hacer para mejorar los valores encontrados.

Por tales razones se requiere identificar amenazas y estrategias de conservación de los servicios ecosistémicos y a su vez genere una amplia gama de acciones para lograr la conservación de la biodiversidad, adecuadas a los contextos locales y que respondan a objetivos claros y metas alcanzables, lo que se ha definido como estrategias de conservación [20].

En este estudio, se logró identificar por medio del mapeo con la participación de los agricultores para cada una de las fincas aquellos lotes en los cuales se presentan tanto amenazas como fortalezas en la generación de los SE.

En el caso de las amenazas para el S.E.R Formación y Regeneración del suelo, se identificaron aquellos lotes con el suelo compacto y polvoriento, con presencia de encharcamiento y de humedad. Con respecto al S.E.R Regulación Climática, se identificaron los lotes en los cuales se evidencia la presencia de vendavales, y con respecto al SER Regulación de las enfermedades, se detectaron aquellos lotes en los cuales hay manifiesta presencia de picudo, plagas y enfermedades.

Con respecto a las fortalezas, para el S.E.A Producción de alimentos, se identificaron los lotes en los cuales el agricultor muestra preferencia para sembrar, donde se logra la mayor producción y se dan los racimos más grandes. Para el S.E.A Actividad biológico se detectaron los lotes donde hay mayor presencia de escarabajos, lombrices y tijeretas. Esto hechos son de gran relevancia en el conocimiento de las condiciones de las fincas, pues permitió detectar hechos, variables e indicadores que se transforman, se modifican y generan cambios en los servicios ecosistémicos; alcance poco abordado para estudios asociados con sistemas de cultivar plátano y que son un gran aporte para complementar el manejo agronómico y los itinerarios técnicos para la producción de musáceas.

\section{Líneas de acción para fortalecer el manejo y la toma de decisiones de los agricultores}


Para el cumplimiento de este objetivo se requiere identificar amenazas y estrategias de conservación de los servicios ecosistémicos, complementado con la generación de una amplia gama de acciones para lograr la conservación de la biodiversidad, adecuadas a los contextos locales y que respondan a objetivos claros y metas alcanzables, lo que se ha definido como estrategias de conservación [21].

La amenaza, es un factor del riesgo, compromete la seguridad de las personas y su medio (asentamientos humanos, infraestructura y unidades productivas) [22].

Con el fin de reducir las amenazas que se presentan para los S.E.R y fortalecer los beneficios en los S.E.A se propuso consolidar el siguiente plan de acción: (i) socializar con cada uno de los agricultores la información obtenida por medio del mapeo participativo, indicando en cuáles lotes de sus fincas se presentaron variaciones en los indicadores evaluados; para luego (ii) programar cursos de intercambio de información que permitan la construcción de soluciones y, (iii) establecer acciones necesarias para la prevención, control, manejo técnico y económico de los cultivos, en armonía con la conservación de los SE, (iv) sensibilizar a los agricultores acerca de la importancia de implementar técnicas de manejo agronómico para mejorar la productividad de los cultivos.

En las líneas de acción también será de gran importancia lograr disminuir las cantidades de fertilizantes y plaguicidas aplicados, evitar que el suelo de los alrededores permanezca desnudo; para lo cual es clave conservar y manejar con rotaciones el crecimiento y las posibilidades de cubrimiento alternado de la superficie del suelo por parte de la vegetación arvense, de acuerdo con las variaciones de las temporadas de lluvias y de sequía.

Promover el uso de los biofertilizantes que son insumos de origen biológico que se aplican al suelo para mejorar la disponibilidad de nutrientes, favoreciendo el desarrollo y el rendimiento de los cultivos y la estabilidad de los agroecosistemas y del ambiente [23].

En el control biológico de plagas o uso de bioplaguicidas utilizar enemigos naturales o competidores vivos como ácaros, caracoles, vertebrados, plantas, virus, bacterias, hongos, nematodos, insectos y otras entidades bióticas inocuas, como también el uso de trampas con vástago para combatir los picudos, con el fin de mantener la densidad de población de un organismo plaga a un nivel que no cause daños importantes y que permita mantener la sustentabilidad del agroecosistema [24].

Además, es importante la instrumentación de sistemas de cultivo integrado, los cuales posibilitan el uso de los recursos propios y disminuye la dependencia de insumos externos en aras de beneficiar la calidad del producto final, disminuir el impacto ambiental generado por la actividad productiva y sin comprometer la seguridad económica de los agricultores y sus familias. Este tipo de sistema involucra además alternativas de control integrado de plagas y enfermedades, las cuales benefician la salud del cultivo y favorecen la dinámica natural del suelo al reducir la presión ejercida sobre este con productos agroquímicos [25].

Los sistemas de cultivar plátano introducidos de manera homogénea y en cultivos en surco, facilitan el impacto y amenazas para los servicios de Formación y Regeneración del suelo, Regulación Climática y de enfermedades; procesos que están asociados con la falta de vegetación acompañante, lo que vulnera la calidad física, química y biológica de los suelos, facilita el mayor impacto de los vendavales y deprime la oferta de servicios ecosistémicos que contribuyan con la sostenibilidad de los sistemas. En estos casos, las decisiones de los agricultores se limitan a métodos intensivos de manejo para intentar resolver los problemas de su cultivo, lo que altera los servicios de los ecosistemas, de manera que se requiere del desarrollo de estrategias más ajustadas a los contextos locales.

Ante estas situaciones, sería de gran valor dar continuidad a líneas de trabajo que incluyan el acompañamiento de los agricultores en el reconocimiento de métodos novedosos, como los documentados para éste estudio, en el que la toma de decisiones se construya de manera conjunta, con la participación de las familias de los cultivadores, para visualizar cómo evolucionan los procesos de cambio en los métodos de manejo utilizados en los sistemas de cultivares de plátano, desde la escala del lote, pasando por la finca, hasta llegar a los municipios.

\section{CONCLUSIONES}

La combinación de mapeo participativo de SE y el análisis basado en SIG puede facilitar la identificación de áreas de protección prioritarias, como también proporcionar orientación para desarrollar estrategias de manejo de las fincas, tales como la realización de talleres técnicos que aporten al agricultor información acorde con sus necesidades, utilizando recursos didácticos y lenguaje claro para que pueda ponerlos en práctica en sus parcelas con el fin de mejorar sus cultivos. Hay que tener presente que es necesario comprender las situaciones particulares en cada una las fincas, como son las amenazas que se presentan y que atentan contra los servicios ecosistémicos de regulación, y las fortalezas que benefician los servicios ecosistémicos de aprovisionamiento, puesto que sus ecosistemas necesitan estar en funcionamiento acorde a las necesidades y expectativas.

Los resultados arrojados en el mapeo con respecto a los servicios ecosistémicos culturales evidenciaron que la percepción de los agricultores frente a los lugares con los que se identifican están dados por el aprecio que tienen hacia la totalidad de la finca, además, ponen énfasis en las áreas construidas tales como viviendas, áreas para reuniones o lugares para cría de animales. Además, identificaron como importantes, aquellas áreas que incluyen los espacios con vegetación natural, nacimientos de agua o cauce de quebradas. Esto significa que existe una preferencia marcada de los agricultores por cierto lugares de la finca, sin importar si esta fue heredada o comprada. Se resalta también el sentido de 
pertenencia y arraigo en la mayoría de las fincas, factor fundamental en la toma de decisiones frente a los cambios a desarrollar para mejorar integralmente las condiciones de éstas. Se destacó como aspecto común a la mayoría de las fincas, que el origen de la propiedad fue mediante herencia, y sus generaciones de antepasados, han permanecido en ellas por más de un siglo, mientras que otras corresponden con su compra o alquiler.

Las percepciones de los agricultores se pueden representar en mapas, que les permiten visualizar más fácilmente para la toma de decisiones. Se logró identificar espacialmente, para cada una de las fincas, dónde se percibe la prestación de cada uno de los $\mathrm{SE}$ estudiados, al igual de dónde se ve reducido. Así mismo, se pudieron asociar las correspondencias entre SE como sinergias e interacciones de forma gráfica lo cual brinda la posibilidad de que los productores determinen qué actividades de campo o estilos de cultivar benefician o perjudican la prestación de los servicios.

\section{REFERENCIAS}

[1] N. Abram, E. Meijaard, M. Ancrenaz, R. Runting, J. Wells, D., Pellier, A. Gaveau, K. Mengersen, "Spatially explicit perceptions of ecosystem services and land cover change in forested regions of Borneo", Ecosystem Services., Vol. 7, pp 116-127. March 2014, DOI: 10.1016/j.ecoser.2013.11.004.

[2] S. Wu, Y. Hou, G. Wan, "Valoración de los bienes y servicios ecosistémicos y del capital forestal natural de la municipalidad de Beijing", XIII Congreso Forestal Mundial, 2010. China. pp. 28-36.

[3] Corporación Colombiana de Investigación Agropecuaria, Corpoica, Manejo sostenible del cultivo del plátano, recuperado en mayo 5 de 2104, http://www.corpoica.org.co/sitioweb/Archivos/Publicaciones/ Cultivo del plátano.pdf, 2006.

[4] P. Lavelle, "Alternativas Biológicas para el Cultivo de Plátano en el Departamento del Quindío, Colombia", Musalac - Bioversity Intertational II Congreso Latinoamericano y del Caribe de Plátano y Banano. 2013. Conf., 21.

[5] J.J. Perfetti,, A. Balcázar, A. Hernández, J. Leibovich, "Políticas para el desarrollo de la agricultura en Colombia 70". 2015.

[6] H. J. Andrade C., M. A. Segura M, E. Sierra R. "Percepción local de los servicios ecosistémicos ofertados en fincas agropecuarias de la zona seca del norte del Tolima, Colombia". Revista Luna Azul, vol. 45, 42-58. 2017. DOI:https://doi.org/10.17151/luaz.2017.45.4

[7] R.S. De Groot, R. Alkemade, L. Braat, L. Hein., L Willemen, "Challenges in integrating the concept of ecosystem services and values in landscape planning, managmemenand decision making" Ecological Complexity. vol 7, Issue 3, September 2010, Pages 260-272 DOI: $10.1016 /$ j.ecocom.2009.10.006.

[8] UNEP-WCMC, "Developing ecosystem service indicators: Experiences and lessons learned from sub-global assessments and other initiatives". Secretariat of the Convention on Biological Diversity, Montréal, Canada. Technical Series No. 58, 118, 2011.

[9] M. C. Zúñiga, A. Feijoo, y H. Quintero, Diseño de una propuesta metodológica para interpretar el huerto habitacional en un área del Valle del Cauca. Scientia et Technica, VOL.2, No. 25, pp. 291-296, 2004.

[10] M. Theriault, C. Claramunt, "Managing time in GIS: an event oriented approach, Recent Advances on Temporal Databases", Eds.: Clifford, J. and A., Tuzhilin, Zurich: Springer-Verlag, Switzerland, 23-42. 1995.

[11] Millennium Ecosystem Assessment, Ecosystem and Human WellBeing: Biodiversity Synthesis, Washington, DC: World Resources Institute, vol. 82. 2005.

[12] O. Masera, M. Asterier, and S. López-Ridaura, "Sustentabilidad y manejo de recursosnaturales. El marco de la evaluación MESMIS". Grupo Interdisciplinario de Tecnología RuralApropiada. Ediciones Mundi Prensa. México. Vol. 1091999.
[13] L. M. F Goodchild, I. Li, "Assuring the quality of volunteered geographic information Spatial Statistics", vol. 1, pp 110-120. 2012 DOI: 10.1016/j.spasta.2012.03.002.

[14] P. Balvanera, y H. Cotler, "Estado y tendencias de los servicios ecosistémicos, en Capital natural de México", pp. 185-245. 2009.

[15] G. Ferrer, F. La Roca, y M. Gual, "Servicios ecosistémicos: ¿Una herramienta útil para la protección o para la mercantilización de la naturaleza?" (2012).

[16] M.A. Altieri, C.I. Nicholls, "Conversión agroecológica de sistemas convencionales de producción: teoría, estrategias y evaluación”. Rev. Ecosistemas. AEET. Universidad de California, Berkeley 16. 2007.

[17] Á. Acevedo, "Metodología para la evaluación de sustentabilidad a partir de indicadores locales para el diseño y desarrollo de programas agroecológicos - MESILPA". Bogotá: Corporación Universitaria Minuto de Dios. Facultad de Ingeniería. 2013.

[18] E. Chango, "Evaluación del avance agroecológico mediante indicadores de sustentabilidad en las fincas de la unión de organizaciones productoras agroecológicas y comercialización asociativa Pacat. Tesis de Magíster en Agroecología y Ambiente". Universidad Técnica de Ambato. Ecuador. 2014.

[19] CE. Fernández, R. Muschler, "Aspectos de sostenibilidad de los sistemas de cultivo de café en América Central. En: Desafíos de la Caficultura en Centro América". Bertrand. B. Ed. San José, Costa Rica. IICA-PROMECAFE-CIRAD. 1999.

[20] S.J. Sarandón, M.S. Zuluaga, R. Cieza, C. Gómez, L. Janjetic, E. Negrete, "Evaluación de la sustentabilidad de sistemas agrícolas de fincas en Misiones", Argentina, mediante el uso de indicadores. En Agroecología 1 (Sarandón SJ,ed.) Ediciones Científicas Americanas. pp. 19-28. 2004.

[21] I.J. March, M.A. Carvajal, R.M. Vidal, J.E. San Román, G. Ruiz et al. "Planificación y desarrollo de estrategias para la conservación de la biodiversidad, en Capital natural de México, vol. II: Estado de conservación y tendencias de cambio". Conabio, México, pp. 545-573. 2009.

[22] M. Cárdenas, P. Choquevillca, J. P. Saavedra, G. Torrico, J. Espinoza, "Construcción de Mapas de Riesgo. Criterios metodológicos". 1. ed. La Paz, BO. 50. 2008.

[23] A. Alarcón, R. Ferrera, "Biofertilizantes: importancia y utilización en la agricultura". Agricultura Técnica en México, vol, 26, No2, pp. 191203. 2000.

[24] C. García-Gutiérrez, y G. D. Rodríguez-Meza. "Problemática y riesgo ambiental por el uso de plaguicidas en Sinaloa". Ra Ximhai. Vol. 8, 3, 1-10. 2012.

[25] L. F. Zuluaga, "Interacciones, compensaciones y sinergias entre servicios ecosistémicos, Eje Cafetero colombiano". Universidad Tecnológica de Pereira. Trabajo de pregrado Administración Ambiental. 2017.

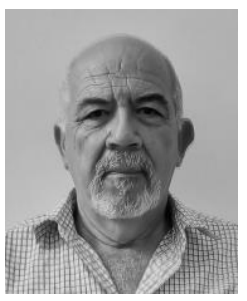

Alberto Javier Botero Arango, nacido el 4 de enero de 1947 en Pereira, Risaralda, Colombia. Ingeniero Industrial egresado de la Universidad Tecnológica de Pereira, Risaralda. Trabajó en Nestlé de Colombia desde el 16 de agosto de 1971 hasta el 16 de agosto del 2001. Magister en Ecotecnologia, Facultad de Ciencias Ambientales, Universidad Tecnológica de Pereira. Actualmente Profesor Asistente de la Facultad de Ciencias Empresariales, Universidad Tecnológica de Pereira. ORCID: https://orcid.org/0000-0003-2123-4183 


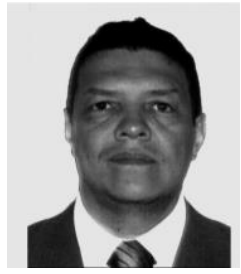

Alexander Feijoo Martínez, nacido el 7 de junio de 1963 en Palmira, Valle del Cauca. Zootecnista, Doctor en Ciencias Agropecuarias de la Universidad Nacional de Colombia, Sede Palmira. Actualmente Profesor Investigador Senior, Titular, Facultad de Ciencias Ambientales, Universidad Tecnológica de Pereira.

ORCID: https://orcid.org/0000-0002-5226-5233

Ligia Janneth Molina Rico, nacida el 19 de noviembre de 1972 en Bogotá D.C., Cundinamarca. Licenciatura en Biología, Universidad Pedagógica Nacional, Magister Universidad Nacional de Colombia Sede Bogotá, Doctora en Ciencias Ambientales de la Universidad Tecnológica de Pereira. Actualmente Profesora Asociada Universidad del Quindío.

ORCID: https://orcid.org/0000-0003-2801-2380

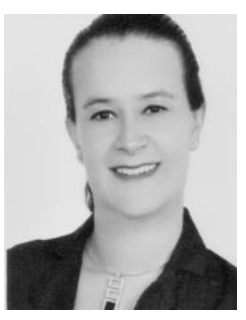

Heimar Quintero Vargas, nacido el 29 de diciembre de 1949 en Roldanillo, Valle del Cauca. Ingeniero Agrónomo de la Universidad Nacional de Colombia, Sede Palmira. Actualmente Profesor Nacional de Colombia.

Pensionado de la Universidad ORCID: https://orcid.org/0000-0003-4548-7169 